\title{
The sphecid wasps (Hym. Ampulicidae, Sphecidae \& Crabronidae) of Egypt. Part 2: Checklist
}

\section{Giles Roche}

Lot 44, Taman Wong Wo Lo, Phase 1, Jalan Tun Mustapha, 87008 W.P. Labuan, MALAYSIA (email: cgroche172@hotmail.com)

\begin{abstract}
The list of Egyptian sphecid wasps includes the current names of those species recorded in the literature with indications where these have changed together with previously unrecorded genera and species. Three genera have not been recorded from Egypt hitherto (Spilomena, Crossocerus, Lestica). Twenty-four species are recorded for the first time (Chalybion heinii, Sceliphron madraspatanum tubifex, Sphex lanatus, Sphex libycus, Prionyx judaeus, Ammophila djaouk, Diodontus brachycerus, Spilomena sp., Astata boops, Dryudella beaumonti, Liris pictipennis, Solierella compedita, Miscophus pseudomimeticus, Belomicrus dimorpha, Belomicrus odontophorus, Oxybelus dissectus, Oxybelus haemorrhoidalis, Crossocerus adhaesus, Lestica clypeata, Bembix joeli, Cerceris clytia, Cerceris lunata, Cerceris rybyensis, Cerceris sabulosa algirica).
\end{abstract}

\section{INTRODUCTION}

This checklist forms part of a series of papers (Roche, 2007a,b; Roche \& Gadallah, 1999; Roche \& Zalat, 1994) dealing with the Egyptian sphecid wasps, of which the generic key (Roche \& Gadallah, 1999) and Sinai list (Roche \& Zalat, 1994) have already been published. The last complete checklist of the sphecid wasps of Egypt was that of Honoré (1942) which also included species of neighbouring countries which could perhaps be found in Egypt.

This present list is based on a search of the literature, augmented by some species found in collections. It excludes some names given in the literature which are nomina dubia and nomina nuda, together with some where it is now believed that the species were recorded from Egypt in error. Where only the nominotypical subspecies occurs in Egypt, the subspecific name is omitted. New species or undetermined material is not mentioned here, with the exception of Spilomena sp. which is mentioned merely to provided a record of the presence of the genus.

Some species recorded in the older literature have never been found in recent collecting and must be considered doubtful records - they may well have been recorded mistakenly based on faulty identification, but it could be that the species concerned did occur but are now extinct in Egypt or that they still linger on but are extremely rare. These species are indicated in the list below with "*" before the name.

Another large group of records concerns species where the name has changed; sometimes this is because research in recent years has amended the generic status of the species; sometimes synonymies and homonymies have been corrected; others cases exist because the species recorded has now been found to comprise more than one species, one or both occurring in Egypt. To assist the use of the basic literature concerning the sphecids of Egypt largely dating from the 1930's and 1940's until new keys and descriptions are available, changes in the genus are indicated by "G" and where the specific name has changed this is indicated by "S". Species not previously recorded for Egypt are shown preceded by "\#" and a number indicating the collection in which they have been found.

The classification used is that of the World Catalog [www.calacademy.org] by Pulawski (2007).

2 = Alfieri collection, El Azhar University Museum, Cairo, Egypt

3 = Mochi collection, Regionale de Scienze Naturali, Torino, Italy

4 = Roche collection, Maidstone Museum, Kent, UK

* [Editor's note: We would like to thank Dr WJ Pulawski for his great help in reorganising this checklist so that it conforms to the latest ideas in sphecid classification (see www.calacademy.org) ] 
Sphecid wasps

(405 species, 6 extra subspecies)

AMPULICIDAE

(1 species)

AMPULICINAE

(1 species)

Dolichurini

(1 species)

Dolichurus haemorrhous A. Costa

SPHECIDAE

AMMOPHILINAE
(60 species, 1 extra subspecies)

(32 species, 1 extra subspecies)
$\# 1$

Ammophila barbara (Lepeletier) ssp. judaeorum Kohl Ammophila djaouak de Beaumont Ammophila dubia Kohl Ammophila erminea Kohl Ammophila gracillima Taschenberg Ammophila guichardi de Beaumont Ammophila haimatosoma Kohl ssp. haimatosoma (s.s.) Ammophila haimatosoma Kohl ssp. sinaitica Alfieri Ammophila hemilauta Kohl Ammophila honorei Alfieri Ammophila horni Schulthess Ammophila insignis F. Smith ssp. egregia Mocsáry Ammophila mitlaensis Alfieri

s Ammophila nasuta Lepeletier ssp. quadraticollis A. Costa Ammophila poecilocnemis Morice Ammophila producticollis Morice

s Ammophila pseudonasuta Bytinski-Salz

$s \quad$ Ammophila rubripes Spinola Ammophila strumosa Kohl

Eremochares dives (Brullé)

Parapsammophila algira (Kohl)

Parapsammophila cyanipennis (Lepeletier)

Parapsammophila dolichostoma (Kohl)

Parapsammophila lateritia Taschenberg

Parapsammophila turanica F. Morawitz

Podalonia affinis (W. Kirby) ssp. affinis (s.s.)

Podalonia atrocyanea (Eversmann) ssp. masinissa (Morice)

Podalonia dispar (Taschenberg)

Podalonia ebenina (Spinola)

Podalonia marismortui (Bytinski-Salz)

Podalonia minax (Kohl)

Podalonia schmiedeknechtii (Kohl)

Podalonia tydei (Le Guillou) ssp. argentata (Lepeletier)

\section{CHLORIONTINAE}

(3 species)

Chlorion funereum Gribodo

Chlorion hirtum (Kohl)

Chlorion semenowi F. Morawitz ssp. occidentale (de Beaumont)

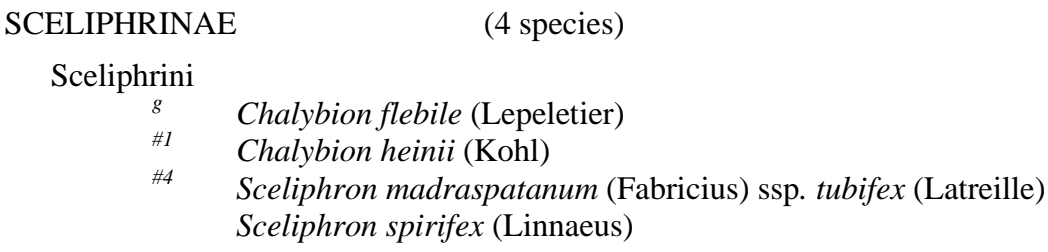

SPHECINAE

Prionychini
(21 species)

(14 species)

Palmodes occitanicus (Lep. \& Serv.) ssp. cyrenaicus (Gribodo) 


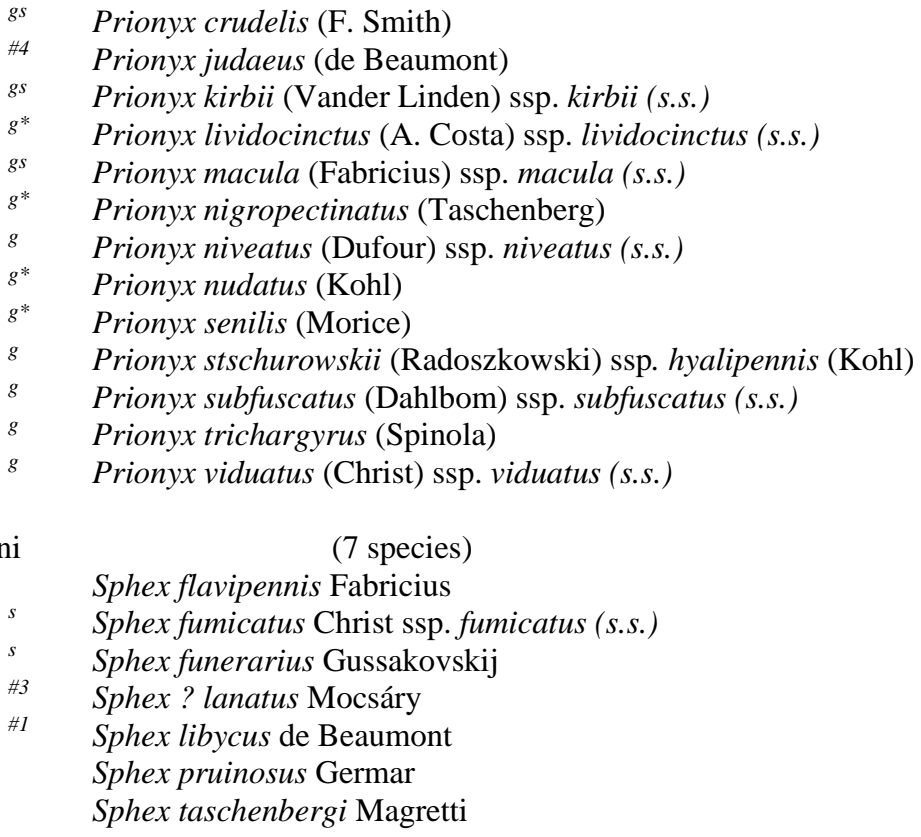

\section{CRABRONIDAE}

\section{(344 species, 6 extra subspecies)}

ASTATINAE

\section{(15 species)}

\#4 Astata boops (Schrank) ssp. boops (s.s.)

Astata cleopatra Pulawski

* Astata fumipennis E. Saunders

* Astata laeta E. Saunders

$s \quad$ Astata lubricata Nurse

* Astata radialis E. Saunders Diploplectron palearcticum Pulawski

$g$ Dryudella amenartais (Pulawski)

$g$ Dryudella ammochtona (Pulawski)

\#4 Dryudella beaumonti (Pulawski)

$g$ Dryudella bifasciata (Schulthess)

$g$ Dryudella elbae (Pulawski)

$g$ Dryudella nephertiti (Pulawski)

$g$ Dryudella opaca (Pulawski)

$g$ Dryudella osiriaca (Pulawski)

DINETINAE (4 species)

Dinetus cereolus Morice

Dinetus dentipes E. Saunders

Dinetus nabateus de Beaumont

Dinetus pulawskii de Beaumont

PEMPHREDONINAE (9 species)

Pemphredonini (7 species)

\#3 Diodontus crassicornis Gribodo

Diodontus clarus Pulawski

Diodontus hyalipennis Kohl

* Diodontus longicornis de Beaumont

Diodontus oraniensis (Lepeletier) ssp. oraniensis (s.s.)

$g$ Protostigmus sinaiticus (Mochi)

\#3 Spilomena sp.

Psenini (2 species)

Mimesa aegyptiaca Radoszkowski

Mimesa sublaevis (de Beaumont) 


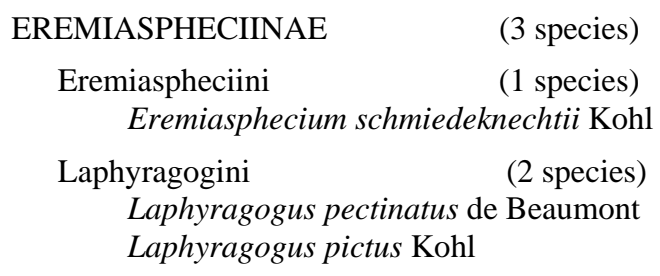

\section{CRABRONINAE}

Larrini

Gastrosericus drewseni (Dahlbom)

$s$ Gastrosericus funereus Gussakovskij

Gastrosericus guigliae de Beaumont

Gastrosericus marginalis Gussakovskij

Gastrosericus moricei E. Saunders

s Gastrosericus pnepheros Pulawski

Gastrosericus thoth Pulawski

Gastrosericus waltlii Spinola

$g$ Holotachysphex holognathus (Morice)

Larra anathema (Rossi)

Liris agilis (F. Smith)

Liris atratus (Spinola)

Liris braueri Kohl

Liris cleopatra de Beaumont

Liris haemorrhoidalis (Fabricius)

$g \quad$ Liris memnonius (F. Smith) ssp. memnonius (s.s.)

$g$ Liris miscophoides Arnold ssp. aegyptiacus de Beaumont

${ }^{g s}$ Liris niger (Vander Linden) ssp. niger (s.s.)

gs Liris nigricans (Walker) ssp. nigricans (s.s.)

$g$ Liris opalipennis (Kohl) ssp. opalipennis (s.s.)

\#4 Liris pictipennis (Maidl)

${ }^{g s}$ Liris festinans (F.Smith) praetermissus (Richards)

$g$ Liris solstitialis (F. Smith) ssp. anubis de Beaumont

$g \quad$ Liris subfasciatus (Walker)

s Parapiagetia erythropoda (Cameron) Parapiagetia genicularis (F. Morawitz)

Parapiagetia krombeini Pulawski

$s$ Parapiagetia mongolica (F. Morawitz)

Parapiagetia odontostoma (Kohl)

Prosopigastra creon (Nurse)

s Prosopigastra fumipennis Gussakovskij

gs Prosopigastra globiceps (F. Morawitz)

Prosopigastra handlirschi Morice

s Prosopigastra insignis E. Saunders

gs Prosopigastra major (F. Morawitz)

$s$ Prosopigastra menelaus (Nurse)

Prosopigastra nuda (Nurse)

Prosopigastra punctatissima A. Costa ssp. africana de Beaumont

Prosopigastra zalinda de Beaumont

\#4 Tachysphex ?aemulus Kohl (needs confirmation)

Tachysphex albocinctus (Lucas)

Tachysphex anubis Pulawski

$s$ Tachysphex argentatus Gussakovskij

s Tachysphex brevipennis Mercet

Tachysphex buyssoni Morice

Tachysphex cheops de Beaumont

Tachysphex chephren de Beaumont

s Tachysphex consocius Kohl ssp. consocius (s.s.)

Tachysphex costae (De Stefani) ssp. costae (s.s.)

Tachysphex crocodilus Pulawski 
Tachysphex denisi de Beaumont

Tachysphex deserticola de Beaumont

$s$ Tachysphex desertorum F. Morawitz

s Tachysphex erythrophorus Dalla Torre

$s$ Tachysphex erythropus (Spinola)

Tachysphex fasciatus Morice

s Tachysphex fugax (Radoszkowski) ssp. fugax (s.s.)

Tachysphex geniculatus (Spinola)

Tachysphex gracilicornis Mercet

Tachysphex grandissimus Gussakovskij

s Tachysphex gujaraticus Nurse

Tachysphex helveticus Kohl ssp. aegyptiacus Morice

$s$ Tachysphex horus de Beaumont

$s$ Tachysphex incertus (Radoszkowski) ssp. nattereri Kohl

s Tachysphex inextricabilis Pulawski

Tachysphex isis de Beaumont

s Tachysphex julliani Kohl

Tachysphex longipalpis de Beaumont ssp. longipalpis (s.s.)

s Tachysphex luxuriosus Morice

Tachysphex detritus Arnold

Tachysphex mediterraneus Kohl

Tachysphex melas Kohl ssp. eatoni E. Saunders

Tachysphex micans (Radoszkowski)

Tachysphex mycerinus de Beaumont

Tachysphex niloticus Pulawski

Tachysphex nitidior de Beaumont

Tachysphex nitidissimus de Beaumont

s Tachysphex nitidus (Spinola) ssp. ibericus (Saussure)

Tachysphex osiris de Beaumont

Tachysphex palopterus (Dahlbom)

Tachysphex panzeri (Vander Linden)

Tachysphex persa Gussakovskij ssp. persa (s.s.)

Tachysphex persa Gussakovskij ssp. catharinae Pulawski

Tachysphex plicosus (A. Costa)

$s$ Tachysphex pompiliformis (Panzer)

Tachysphex priesneri de Beaumont

Tachysphex ptah Pulawski

Tachysphex ramses Pulawski

Tachysphex schmiedeknechti Kohl

Tachysphex sericans Gussakovskij ssp. gracilis Pulawski

Tachysphex speciosissimus Morice

Tachysphex tarsinus (Lepeletier)

$s$ Tachysphex unicolor (Panzer)

Tachysphex vestitus Kohl

Tachysphex yarrowi de Beaumont

Tachytes alfierii Pulawski

$s$ Tachytes archaeophilus Pulawski

$s$ Tachytes argyreus (F. Smith)

Tachytes basilicus Guérin-Méneville

Tachytes biskrensis E. Saunders

Tachytes brunneus Pulawski

Tachytes cameronianus Morice

s Tachytes comberi Turner

Tachytes diodontus Pulawski

s Tachytes diversicornis Turner

s Tachytes fucatus Arnold

Tachytes levantinus Pulawski

Tachytes maculicornis E. Saunders

$s$ Tachytes maroccanus Pulawski

Tachytes niloticus Turner

Tachytes nomarches Pulawski 
Tachytes obsoletus (Rossi) ssp. obsoletus (s.s.)

Tachytes obsoletus (Rossi) ssp. tricoloratus (Turton)

Tachytes priesneri Pulawski

s Tachytes pygmaeus Kohl

Tachytes tarsalis (Spinola)

Tachytes trichopygus Pulawski

Palarini (8 species)

s Palarus ambustus Klug ssp. ambustus (s.s.)

$s$ Palarus dongalensis Klug

Palarus hastatifrons Turner ssp. africanus de Beaumont

Palarus histrio Spinola

s Palarus laetus Klug ssp. laetus (s.s.)

Palarus parvulus de Beaumont

Palarus saundersi Morice

Palarus spinolae Saussure ssp. spinolae (s.s.)

Miscophini

s Miscophus aegyptius Morice

Miscophus aenigma Honoré

Miscophus affinis Pulawski

s Miscophus alfierii Honoré

Miscophus bicolor Jurine

Miscophus clypearis Honoré

Miscophus collaris Honoré

s Miscophus ctenopus Kohl

Miscophus dispersus de Andrade

Miscophus flavopictus Pulawski

Miscophus funebris Honoré

Miscophus imitans Giner Marí

Miscophus inconspicuus de Andrade

Miscophus mimeticus Honoré

s Miscophus mochii Arnold

s Miscophus nigripes Honoré Miscophus niloticus Honoré Miscophus obscuritarsis Pulawski

Miscophus papyrus de Andrade

$s$ Miscophus pharaonis Arnold

\#4 Miscophus pseudomimeticus de Andrade

s Miscophus sericeus Radoszkowski

Miscophus similis F. Morawitz

Nitela mochii Arnold

Solierella aegyptia Kohl

\#4 Solierella ?compedita (Piccioli) ssp. compedita (s.s.)

Solierella dispar Pulawski

Solierella longicornis Pulawski

Solierella nigridorsum Pulawski

Solierella nitraria Pulawski

Solierella pectinata Pulawski

Trypoxylonini (5 species)

s Pison carinatum Turner

Trypoxylon aegyptium Kohl

* Trypoxylon albipes F. Smith

* Trypoxylon attenuatum F. Smith ssp. attenuatum (s.s.)

* Trypoxylon scutatum Chevrier

Oxybelini (18 species)

4 Belomicrus dimorpha Guichard

Belomicrus mirificus Kohl

\#4 Belomicrus odontophorus (Kohl)

Belomicrus pharaonum Pulawski

Oxybelomorpha waterstonii Kohl 
Oxybelus aurantiacus Mocsáry

Oxybelus collaris Kohl

* Oxybelus continuus Dahlbom

Oxybelus diphyllus (A. Costa) ssp. pharao Kohl

\#4 Oxybelus dissectus Dahlbom

$s$ Oxybelus fischeri Spinola ssp. fischeri (s.s.)

Oxybelus guichardi de Beaumont

\#4 Oxybelus haemorrhoidalis Olivier

Oxybelus lamellatus Olivier ssp. lamellatus (s.s.)

Oxybelus palmetorum de Beaumont

Oxybelus phyllophorus Kohl

* Oxybelus subspinosus Klug

*g Pseudomicroides santschii (Schulthess)

Crabronini

(10 species)

\#4 Crossocerus adhaesus (Kohl)

$g$ Dasyproctus arabs (Kohl)

$g$ Ectemnius confinis (Walker)

$g$ Ectemnius continuus (Fabricius)

${ }^{*}$ Ectemnius hypsae (De Stefani)

${ }^{* g}$ Entomognathus brevis (Vander Linden)

\#4 Lestica clypeata (Schreber)

Lindenius aegyptius (Kohl)

Lindenius difficillimus (Kohl)

Lindenius haemodes (Kohl)

BEMBECINAE (74 species, 3 extra subspecies)

Nyssonini

(2 species)

Nysson laevis Pulawski

Nysson rufus Handlirsch

Bembecini (72 species, 3 extra subspecies)

$g$ Ammatomus coarctatus (Spinola)

$g$ Ammatomus mesostenus (Handlirsch)

$g$ Bembecinus tenellus (Klug)

* Bembecinus argentifrons F.Smith

Bembix abragensis Priesner

Bembix alfierii Priesner

Bembix arenaria Handlirsch

$s$ Bembix capensis Lepeletier

Bembix chlorotica Spinola

Bembix chopardi Berland

Bembix dahlbomii Handlirsch ssp. dahlbomii (s.s.)

Bembix fischeri Spinola ssp. fischeri (s.s.)

Bembix flavescens F. Smith ssp. kittyae de Beaumont

Bembix flavescens F. Smith ssp. picturata Bytinski-Salz

Bembix freygessneri Morice

\#4 Bembix joeli Bytinski-Salz

Bembix kohlii Morice

Bembix lusca Spinola

* Bembix maidli Schulthess

Bembix melanura F. Morawitz

Bembix nasuta Morice

Bembix nilotica Priesner

Bembix oculata Panzer ssp. soror Dahlbom

Bembix olivacea Fabricius ssp. saharae Giner Marí

Bembix palaestinensis Lohrmann

Bembix pallescens Priesner

* Bembix portschinskii Radoszkowski

Bembix priesneri de Beaumont

Bembix radoszkowskii Handlirsch 
Bembix rufiventris Priesner

s* Gorytes foveolatus Handlirsch ssp. longicornis Handlirsch

* Gorytes sulcifrons (A. Costa)

g Harpactus castor (Handlirsch)

$g$ Harpactus laevis (Latreille) ssp. aegyptiacus (Schulz)

${ }^{* g}$ Harpactus lunatus (Dahlbom)

${ }^{* g}$ Harpactus pollux (Handlirsch)

$g$ Hoplisoides ferrugineus (Spinola)

Kohlia coxalis Morice

Psammaecius eremorum de Beaumont Sphecius claripennis Morice

$g \quad$ Stizoides abdominalis (Dahlbom)

$g \quad$ Stizoides assimilis (Fabricius)

$g$ Stizoides citrinus (Klug)

${ }^{* g}$ Stizoides crassicornis (Fabricius)

$g \quad$ Stizoides klugii (F. Smith) Stizoides melanopterus (Dahlbom)

$g$ Stizoides tridentatus (Fabricius)

* Stizus aegyptius Lepeletier

* Stizus annulatus (Klug) Stizus arnoldi Mochi

* Stizus basalis Guérin-Méneville Stizus bizonatus Spinola

$s$ Stizus combustus (F. Smith)

* Stizus elegans Dahlbom Stizus fuscatus Morice Stizus hyalipennis Handlirsch

s Stizus lepidus (Klug)

$s$ Stizus marthae Handlirsch Stizus niloticus Handlirsch ssp. niloticus (s.s.) Stizus niloticus Handlirsch ssp. saharae Roth

* Stizus pictus Dahlbom

* Stizus rapax Handlirsch Stizus ruficornis (J. Forster) ssp. ruficornis (s. s.)

Stizus ruficornis (J. Forster) ssp. strigatus Mochi

* Stizus rufiventris Radoszkowski ssp. compar Handlirsch

* Stizus rufocinctus Dahlbom

* Stizus rufoniger Mochi

* Stizus savignyi Spinola

* Stizus schmiedeknechti Handlirsch Stizus spinulosus Radoszkowski Stizus tricolor Handlirsch

* Stizus tunetanus A. Costa Stizus vespoides (Walker)

* Stizus zonatus (Klug)

* Stizus zonosoma Handlirsch

PHILANTHINAE (52 species, 1 extra subspecies)

Philanthini (8 species, 1 extra subspecies)

Philanthus triangulum (Fabricius) ssp. abdelcader Lepeletier

* Philanthus triangulum (Fabricius) ssp. obliteratus Pic

Philanthus genalis Kohl

Philanthus rutilus Spinola

Philanthus coarctatus Spinola

Philanthus minor Kohl

Philanthus pallidus Klug

* Philanthus venustus (Rossi)

$s \quad$ Philanthus variegatus Spinola ssp. variegatus (s.s.)

Aphilanthopsini (1 species)

Philanthinus integer (de Beaumont) 


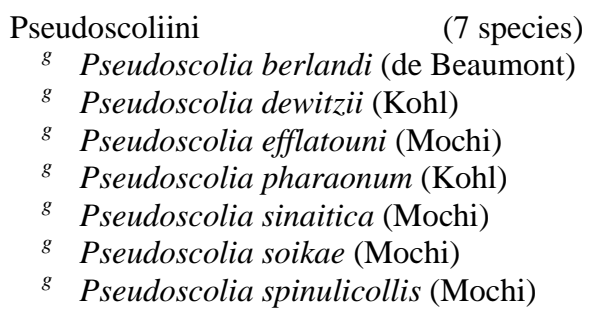

Cercerini

Cerceris albicincta Klug

(36 species)

$s$ Cerceris alboatra Walker

Cerceris berenice de Beaumont

Cerceris bupresticida Dufour ssp. libyca de Beaumont

$s$ Cerceris cheops de Beaumont

$s$ Cerceris chlorotica Spinola

$s$ Cerceris chromatica Schletterer

\#4 Cerceris clytia de Beaumont

Cerceris fimbriata (Rossi) ssp. fimbriata (s.s.)

$s$ Cerceris fischeri Spinola

$s$ Cerceris hathor Pulawski

gs Cerceris histerisnica (Spinola)

s Cerceris histrionica Klug

Cerceris klugii F. Smith

Cerceris lateriproducta Mochi

\#4 Cerceris lunata A. Costa

$s$ Cerceris luxuriosa Dahlbom

Cerceris margarita de Beaumont

Cerceris pallidula Morice ssp. pallidula (s.s.)

Cerceris palmetorum de Beaumont

Cerceris pharaonum Kohl

Cerceris priesneri Mochi

Cerceris pruinosa Morice

s Cerceris pulchella Klug ssp. pulchella (s.s.)

* Cerceris quadricincta (Panzer) ssp. quadricincta (s.s.) Cerceris rhinoceros Kohl

Cerceris rutila Spinola ssp. rutila (s.s.)

\#4 Cerceris rybyensis (Linnaeus)

\#4 Cerceris sabulosa (Panzer) ssp. algirica (Thunberg)

s Cerceris sinaitica de Beaumont

$s$ Cerceris solitaria Dahlbom

* Cerceris specularis A. Costa ssp. specularis (s.s.)

$s$ Cerceris spinipectus F. Smith ssp. spinolica Schletterer

$s$ Cerceris straminea Dufour ssp. komarovii Radoszkowski

Cerceris tyrannica F.Smith

$s$ Cerceris tricolorata Spinola

\section{References}

Bohart RM \& Menke AS (1976) Sphecid wasps of the world: a generic revision. University of California Press, 695pp Honoré AM (1942) Introduction à l'étude des Sphégides en Egypte. Bulletin de la Société Fouad 1er d'Entomologie 26: 25-80. Roche GC (2007a) Then and Now. Egyptian Journal of Natural History 4:

Roche GC (2007b) Conspectus of the Sphecid wasps of Egypt (Hymenoptera: Sphecidae). Egyptian Journal of Natural History 4

Roche GC \& Gadallah NS (1999) The sphecid wasps of Egypt (Hymenoptera: Sphecidae): Introduction and generic key. Egyptian Journal of Biology 1: 104-117.

Roche GC \& Zalat SM (1994) The Sphecidae (Hymenoptera) of the Sinai Peninsula. pp. 113-118 in Proceedings of the $1^{\text {st }}$ Egyptian-Hungarian Conference on Environment, St Catherine, Sinai, Egypt, April 5-7 ${ }^{\text {th }}$, 1993. Suez Canal University, Ismailia. 323 pp. 